\title{
The Risk of Febrile Seizures Following Influenza and 13-Valent Pneumococcal Conjugate Vaccines
}

\section{Citation}

Baker, M., C. Jankosky, K. Yih, S. Gruber, L. Li, N. Cocoros, H. Lipowicz, et al. 2017. "The Risk of Febrile Seizures Following Influenza and 13-Valent Pneumococcal Conjugate Vaccines." Open Forum Infectious Diseases 4 (Suppl 1): S464-S465. doi:10.1093/ofid/ofx163.1187. http:// dx.doi.org/10.1093/ofid/ofx163.1187.

\section{Published Version}

doi:10.1093/ofid/ofx163.1187

\section{Permanent link}

http://nrs.harvard.edu/urn-3:HUL.InstRepos:34493265

\section{Terms of Use}

This article was downloaded from Harvard University's DASH repository, and is made available under the terms and conditions applicable to Other Posted Material, as set forth at http:// nrs.harvard.edu/urn-3:HUL.InstRepos:dash.current.terms-of-use\#LAA

\section{Share Your Story}

The Harvard community has made this article openly available.

Please share how this access benefits you. Submit a story.

\section{Accessibility}


1488. Post-licensure Surveillance of 13-Valent Pneumococcal Conjugate Vaccine (PCV13) in Children 6 weeks-59 months old, Vaccine Adverse Event Reporting System (VAERS), United States, 2010-2017

Jorge Arana, $\mathrm{MD}, \mathrm{MPH}^{1}$; Pedro Moro, $\mathrm{MD}, \mathrm{MPH}^{2}$; Paige Lewis, $\mathrm{MSPH}^{2}$ and Maria Cano, MD, $\mathrm{MPH}^{3}$; ${ }^{1}$ Immunization Safety Office, Centers for Disease Control and Prevention (CDC), Atlanta, Georgia, ${ }^{2}$ Immunization Safety Office, Centers for Disease Control and Prevention, Atlanta, Georgia, ${ }^{3}$ Centers for Disease Control and Prevention (CDC), Atlanta, Georgia

Session: 165. Pneumococcal Immunization and Epidemiology-North America Friday, October 6, 2017: 12:30 PM

Background. In February 2010, the Food and Drug Administration (FDA) licensed the 13-valent pneumococcal conjugate vaccine (PCV13) for use in children aged 6 weeks through 59 months. The Advisory Committee on Immunization Practices (ACIP) recommended routine use of PCV13 for all children ages 2, 4, and 6 months with a booster at $12-15$ months. For incomplete or unvaccinated children, catch-up vaccination should occur through age 59 months.

Methods. We searched the Vaccine Adverse Event Reporting System (VAERS), a U.S. passive surveillance system, for reports of adverse events (AEs) following PCV13 from February 24, 2010 through February 24, 2017, in children aged 6 weeks through 59 months. Signs and symptoms of AEs were coded using the Medical Dictionary for Regulatory Activities (MedDRA). Physicians reviewed the VAERS forms and available medical records of serious reports (death, life-threatening illness, hospitalization, prolongation of hospitalization and permanent disability) and reports of Kawasaki disease and anaphylaxis. Cause of death was ascertained by review of death certificate and/or autopsy report.

Results. VAERS received 10,007 reports after PCV13; 1,706 (17.0\%) were serious. In 927 (9.3\%), PCV13 was administered alone. The most frequently reported symptoms were pyrexia $(26.4 \%)$, injection site erythema $(15.3 \%)$ and irritability (14.6\%). Injection site erythema (25.4\%), injection site swelling $(20.6 \%)$ and pyrexia (20.1\%) were most frequent among children who were given PCV13 alone. Median time from vaccination to start of symptoms was 1 day (range: day of vaccination - 2,033 days). There were $222(2.2 \%)$ death reports with sudden infant death syndrome as the most common cause $(37.8 \%)$. Pyrexia (45.1\%), irritability $(40.4 \%)$, and vomiting $(39.7 \%)$ were most commonly reported among non-death serious reports. There were $20(0.2 \%)$ reports of Kawasaki disease and $20(0.2 \%)$ reports of anaphylaxis.

Conclusion. AES reported to VAERS following PCV13 were consistent with AEs previously observed in pre-licensure clinical trials and other post-licensure studies of PCV13. No new or unexpected patterns of AEs were identified.

Disclosures. All authors: No reported disclosures.

\section{Invasive Pneumococcal Disease in a Population with Underlying} Comorbidities

Daniel Jarovsky, MD; Eitan Naaman Berezin, MD and Rodrigo José Sini De Almeida, MD; Pediatric, Santa Casa de Misericórdia de São Paulo, São Paulo, Brazil

Session: 165. Pneumococcal Immunization and Epidemiology-North America Friday, October 6, 2017: 12:30 PM

Background. Streptococcus pneumoniae (Spn) is a major cause of severe and life-threatening diseases in children and particularly among individual with high-risk illnesses at all ages. As there is limited clinical data on IPD in high-risk patients and indirect effect of vaccination in the post-PCV10 era in developing countries, we've assessed the epidemiology of IPD in patients with and without selected underlying diseases before and after PCV10 introduction at Santa Casa de São Paulo (SCSP), Brazil.

Methods. We've performed a prospective hospital-based surveillance study of patients with IPD from January 2000 to April 2017, including all cases of IPD (i.e isolation of $S p n$ from a normally sterile body fluid) among patients at all ages. Selected cases were stratified into 5 age groups to evaluate comorbidities and the effect of the PCV10 on different ages. Identified serotypes were grouped according to the available pneumococcal vaccines and further analyzed into pre-vaccination (2000-2009) and post-vaccination periods (2010-2017). Clinical information was extracted from patient's records, then stratified based on their IPD risk profile. Ethical approvals to conduct the study were obtained from the SCSP institutional review board.

Results. 571 episodes were identified in 561 patients in all age groups, of which $440(78.4 \%)$ had clinical data for analysis: $20.7 \%$ healthy; $79.3 \%$ had comorbidities. IPD decreased from 35.9 to 30.3 cases/year $(-15.6 \%)$ at all ages after PCV10 introduction. Among healthy individuals and those with underlying comorbidities, annual cases changed from 6.8 to 2.9 (57.3\% reduction) and 18.6 to 20.5 cases/year $(9.7 \%$ increase), respectively, between same periods; 30 -day mortality through pre-vaccine period was $25 \%$ and $7.5 \%$ and in post-PCV10 period $27 \%$ and $8.7 \%$, for comorbidity and healthy groups, respectively. IPD significantly decreased among healthy and comorbidity children $<5 y$, without evidence of serotype replacement. Significant increase in bacteremia and pneumonia, also in serotypes included in all vaccines and NVT was evidenced at ages over $5 y$.

Conclusion. High rates of IPD have persisted in older subjects and in patients with established risk factors for IPD, despite children vaccination with PCV10. No herd effect was detected and serotype replacement is ongoing in this specific groups.

Disclosures. E. N. Berezin, Pfizer: Grant Investigator, Educational grant; R. José Sini De Almeida, Pfizer Inc: Employee, Salary
1490. Pneumococcal Vaccination Provides Substantial Value for Money for Canadians

Francois Peloquin, $\mathrm{BSc}^{1}$; Marie-Claude Breton, $\mathrm{MPharm}^{1}$; Matt Wasserman, $\mathrm{MSc}^{2}$; Michele Wilson, $\mathrm{MSPH}^{3}$; Cheryl McDade, $\mathrm{BA}^{3}$ and Raymond Farkouh, $\mathrm{PhD}^{4}$; ${ }^{1}$ Pfizer Canada, Kirkland, QC, Canada, ${ }^{2}$ Pfizer Inc., New York, New York, ${ }^{3}$ RTI Health Solutions, Research Triangle Park, North Carolina, ${ }^{4}$ Pfizer Inc, Collegeville, Pennsylvania

Session: 165. Pneumococcal Immunization and Epidemiology-North America Friday, October 6, 2017: 12:30 PM

Background. Introduction of pneumococcal conjugate vaccines (PCV) to the Canadian childhood routine immunization schedules (RIS) resulted in significant benefits. The 7-valent PCV was added to all provinces' RIS between 2002 and 2006. The 10-valent PCV was used in Ontario and Quebec for 12 to 18 months in 2009 and 2010. The 13-valent PCV was marketed in 2010 and rapidly adopted by all provinces. Direct vaccine protection reduced incidence of invasive pneumococcal disease (IPD), pneumonia (PNE) and acute otitis media (AOM) in vaccinated children. Indirect vaccine protection also reduced the burden of disease (BOD) in other age groups. Sensible public funds allocation motivates continued evaluation of public health programs.

Objective: To evaluate the economic impact of PCVs to Canadian society following nationwide RIS implementation.

Methods. Canadian databases and literature were reviewed to obtain pre- and post-PCV incidence of IPD, PNE and AOM, as well as direct and indirect medical costs (reported in 2017 \$ CAD). Case counting index date was set to Jan 2005, at which point PCV RIS were implemented for over $90 \%$ of Canadians. A steady state scenario using pre-PCV incidence rates was projected to Dec 2015 to estimate the number of cases without PCVs. Averted cases were obtained by subtracting the cases reported from the estimated case count without PCVs. Disease specific costs were assigned to averted cases and vaccine spend was subtracted from the total to obtain net savings to Canadian society.

Results. Successive implementation of PCVs on the provinces' RIS saved 2,365 lives and resulted in net savings of CAD \$203 million between Jan 2005 and Dec 2015. These savings stem from averted direct and indirect medical costs associated with IPD, $\mathrm{PNE}$ and AOM cases.

Table 1 - BOD and related costs avoided by PCV use, 2005-2015

\begin{tabular}{lccc}
\hline & With PCVs & Without PCVs & Difference \\
\hline Case count & 27,041 & 36,808 & \\
Bacteremia & 14,461 & 19,685 & $-9,767$ \\
Meningitis & 366,927 & 386,413 & $-5,223$ \\
Hospitalized PNE & 545,230 & 589,251 & $-19,486$ \\
Nonhospitalized PNE & $3,629,952$ & $4,374,467$ & $-44,021$ \\
AOM & $\$ 7,123$ & $\$ 8,078$ & $-744,514$ \\
Costs ( $\$$ million) & $\$ 753$ & $\$ 0$ & $-\$ 955$ \\
Disease related & $\$ 7,876$ & $\$ 8,078$ & $\$ 753$ \\
Vaccine cost & & & $-\$ 203$ \\
Total & 36,917 & 39,282 & $-2,365$ \\
Other (no cost considered) & & & \\
Mortality & &
\end{tabular}

Conclusion. Introduction of PCVs resulted in reduced pneumococcal burden of disease and net economic benefits to Canadian society.

Disclosures. F. Peloquin, Pfizer: Employee, Salary; M. C. Breton, Pfizer: Employee, Salary; M. Wasserman, Pfizer: Employee, Salary; M. Wilson, Pfizer: Consultant, Consulting fee; C. McDade, Pfizer: Consultant, Consulting fee; R. Farkouh, Pfizer: Employee, Salary

1491. The Risk of Febrile Seizures Following Influenza and 13-Valent Pneumococcal Conjugate Vaccines

Meghan Baker, MD, $\mathrm{ScD}^{1,2}$; Christopher Jankosky, MD, MPH ${ }^{3}$; Katherine Yih, $\mathrm{PhD}, \mathrm{MPH}^{1}$; Susan Gruber, $\mathrm{PhD}^{1}$; Lingling Li, $\mathrm{PhD}^{4}$; Noelle Cocoros, DSc, $\mathrm{MPH}^{1}$; Hana Lipowicz, $\mathrm{MPH}^{1}$; Claudia Coronel-Moreno, $\mathrm{MPH}^{1}$; Sandra Feibelmann, $\mathrm{MPH}^{1}$; Nancy Lin, $\mathrm{ScD}^{5}$; Cheryl McMahill-Walraven, PhD, MSW ${ }^{6}$; David Menschik, MD, $\mathrm{MPH}^{3}$; Mano Selvan, $\mathrm{PhD}^{7}$; Nandini Selvam, $\mathrm{PhD}, \mathrm{MPH}^{8}$; Rong Chen Tilney, $\mathrm{MS}^{1}$; Lauren Zichittella, MS ${ }^{1}$; Grace Lee, MD, MPH, FPIDS ${ }^{1,9}$ and Alison Tse Kawai, $\mathrm{ScD}, \mathrm{SM}^{1} ;{ }^{1}$ Department of Population Medicine, Harvard Pilgrim Health Care Institute and Harvard Medical School, Boston, Massachusetts, ${ }^{2}$ Brigham and Women's Hospital, Boston, Massachusetts, ${ }^{3} \mathrm{FDA}$ Center for Biologics Evaluation and Research, Silver Spring, Maryland, ${ }^{4}$ Sanofi Genzyme, Cambridge, Massachusetts, ${ }^{5}$ Optum Epidemiology, Boston, Massachusetts, ${ }^{6}$ Aetna, Blue Bell, Pennsylvania, ${ }^{7}$ Comprehensive Health Insights, Sugar Land, Texas, ${ }^{8}$ QuintilesIMS, Fairfax, VA, ${ }^{9}$ Division of Infectious Diseases, Boston Children's Hospital, Boston, Massachusetts

Session: 165. Pneumococcal Immunization and Epidemiology-North America Friday, October 6, 2017: 12:30 PM

Background. Evidence on the risk of febrile seizures (FS) after vaccination with inactivated influenza vaccine (IIV) and 13-valent pneumococcal conjugate vaccin (PCV13) is mixed. Among children 6-23 months, we examined the risk of FS following IIV and PCV13 during the 2013-14 and 2014-15 influenza seasons, for which vaccine virus strains were the same. 
Methods. We used claims data from 4 large national insurers in the FDAsponsored Sentinel Initiative, which was developed to monitor the safety of FDAregulated medical products. With a self-controlled risk interval design, the risk of FS in $0-1$ days following IIV and following PCV13 was compared with a comparison interval (14-20 days), adjusting for confounding by age, calendar time, and concomitant vaccination with the other vaccine. In exploratory analyses, we assessed whether the effect of IIV is modified by concomitant administration of PCV13.

Results. During the study period, 355,486 children received IIV and 581,868 received PCV13. We observed an incidence rate ratio (IRR) of 1.12 (95\% CI $0.80,1.56)$ for the risk of FS following IIV after adjustment for age, calendar time and concomitant PCV13. PCV13 was associated with an increased risk of FS (IRR adjusted for age, calendar time and concomitant IIV, 1.80, 95\% CI 1.29, 2.52). The attributable risk for PCV13 ranged from 0.33 to 5.16 per 100,000 doses.

The age and calendar-time adjusted IRR comparing exposed time to unexposed time was greater for concomitant IIV and PCV13 (IRR 2.80, 95\% CI 1.63, 4.83), as compared with that for PCV13 without concomitant IIV (IRR 1.54, 95\% CI 1.04, 2.28). However, the formal test assessing for interaction between IIV and PCV13 was not statistically significant.

Conclusion. We found an elevated risk of FS after PCV13 vaccine but not after IIV, when adjusting for concomitant administration of the other vaccine. We found some evidence to suggest that concomitant administration of IIV with PCV13 might interact to increase the risk beyond the independent effects of PCV13, but the study was not powered to assess this interaction. The risk of seizures associated with PCV13 is low compared with a child's lifetime risk of FS. Findings should be interpreted in the context of the importance of preventing influenza and pneumococcal infections in young children.

Disclosures. L. Li, sanofi pasteur: The author is currently employed by Sanof Genzyme, which shares the same parent company as sanofi pasteur, the manufacturer of the Flu vaccine. However, the work was done while this author was still employed by Harvard Pilgrim Health Care Institute., No financial benefit received

1492. Incidence of Invasive Pneumococcal Disease Before and During an Era of Use of Three Different Pneumococcal Conjugate Vaccines in Quebec Philippe De Wals, $\mathrm{MD}, \mathrm{PhD}^{1}$; Brigitte Lefebvre, $\mathrm{PhD}^{2}$; Geneviève Deceunicnk, MSc and Jean Longtin, $\mathrm{MD}^{4} ;{ }^{1}$ Social and Preventive Medicine, Laval University, Quebec City, QC, Canada, ${ }^{2}$ INSPQ, Montreal, QC, Canada, ${ }^{3}$ Quebec University Hospital Research Centre, Quebec City, QC, Canada, ${ }^{4}$ Laboratoire De Santé Publique Du Québec, Inistitut national de santé publique du Québec, Sainte-Anne-de-Bellevue, QC, Canada

Session: 165. Pneumococcal Immunization and Epidemiology-North America Friday, October 6, 2017: 12:30 PM

Background. In Quebec, 7-valent (PCV7), 10-valent (PCV10) and 13-valent (PCV13) pneumococcal conjugate vaccines were successively used for children immunization according to a $2+1$ doses schedule.

Objective: To assess the impact of this program on the epidemiology of invasive pneumococcal disease (IPD).

Methods. Notification (2000-2016) and laboratory surveillance (2005-2016) data were analyzed and the immunization status of IPD cases in 2011-2015 was checked.

Results. In children $<5$ years, IPD rate decreased from 69/100,000 in 2003 to $12 / 100,000$ in 2016 ( $83 \%$ reduction). Following PCV7 introduction in 2004, there was a rapid decline in incidence of homologous serotypes. $7 \mathrm{~F}$ cases and 19A decreased following PCV10 introduction in 2009 and PCV13 in 2011, whereas decrease in serotype 3 IPD was minimal. Non-vaccine types IPD increased and represented $79 \%$ of cases in 2016 . The same pattern was seen in adults but replacement was complete and there was no decrease in overall IPD rate. In those 65 years and over, PCV13 serotypes represented $28 \%$ of cases in 2016 , and $62 \%$ were covered by the 23 -valent polysaccharide vaccine. Out of 7 IPD cases caused by serotype 3 in children vaccinated with PCV13, 5 occurred more than one year following the booster dose, which suggests short-term protection. Out of 27 breakthrough 19A cases, 17 occurred between 8 and 14 months of age in children who had received the 2 primary PCV13 doses but not the toddler booster dose, which suggests a window of susceptibility in a $2+1$ schedule.

Conclusion. Hopefully, 19A incidence in children will continue to decrease and herd protection would eventually close the window of susceptibility. Serotype 3 is fortunately not frequent in children but a hard nut to crack. In elderly adults, PCV13 serotype coverage is diminishing year after year but a majority of cases remains potentially covered by the 23 -valent polysaccharide vaccine.

Disclosures. P. De Wals, GSK: Investigator and Scientific Advisor, Research grant and Travel expenses; Pfizer: Grant Investigator and Scientific Advisor, Research grant and Travel expenses; SanofiPasteur: Grant Investigator, Research grant and Travel expenses

1493. Enhanced Detection of Vaccine Type Colonization and Dual Serotype Carriage with Molecular Strategies for Identification of Streptococcus pneumoniae Colonization

Brent A. Little, $\mathrm{PhD}^{1}$; Julianne Meehan, $\mathrm{MPH}^{2}$; Kimberly M. Shea, $\mathrm{PhD}, \mathrm{MPH}^{3}$ and Stephen I. Pelton, $\mathrm{MD}^{3} ;{ }^{1}$ Pediatric Infectious Diseases, Boston University, Boston,
Massachusetts, ${ }^{2}$ Boston Medical Center, Boston, Massachusetts, ${ }^{3}$ Boston University Schools of Medicine and Public Health, Boston, Massachusetts

Session: 165. Pneumococcal Immunization and Epidemiology-North America Friday, October 6, 2017: 12:30 PM

Background. Detecting Streptococcus pneumoniae (SP) carriage in children via conventional microbiological methods lacks sensitivity as high density is required for routine culture and identification of dual serotype colonization is a technical challenge. To increase understanding of post vaccine nasopharyngeal (NP) carriage, specifically persistence of vaccine serotypes, strategies for molecular identification of SP have been developed. These methods most often rely on the identification of the SP autolysin gene lytA through non-quantitative PCR or semi-quantitative real-time PCR (RTPCR)

Methods. We collected NP swabs from 600 healthy or sick children $<5$ years old at Boston Medical Center from Nov 2015 - Mar 2016 and used enhanced microbiologic culture and molecular identification strategies to characterize SP colonization. NP specimens were broth enriched for 4 hours and cultured on selective blood agar; routine microbiologic methods were used to isolate and identify SP. A second aliquot of enriched broth was used for DNA isolation. RTPCR assays were performed targeting the lytA, piaB (SP membrane permease), cpsA (SP capsule operon) genes, and $21 \mathrm{SP}$ serotypes: all serotypes included in 13-valent pneumococcal conjugate vaccine and 8 additional non-vaccine serotypes.

Results. In our sample, $16 \%$ of specimens were SP positive via culture and $33 \%$ were RTPCR-positive $(\mathrm{Cq} \leq 35)$ for the lytA gene. Multiplex RTPCR assays with both the $l y t A$ and piaB genes yielded a positive result for $24 \%$ of samples. Further RTPCR confirmed that $99 \%$ of the $l y t A / p i a B$ positive samples were positive for $c p s A$ (a second marker for assay validation) but only $70 \%$ of the $l y t A$ positive, paiB negative samples were $c p s A$ positive. Serogroup 19 was the most frequently isolated vaccine serogroup using both culture and RTPCR; molecular analysis identified $6 \%$ of specimens with concurrent carriage of more than one serotype.

Conclusion. Compared with enhanced culture, we found a 50\% increase in SP detection using combined $l y t A$ and piaB multiplex RTPCR. Similarly, the proportion of children colonized with vaccine serotypes increased from $2 \%$ to $7 \%$. This work is funded by an investigator initiated grant to BUMC from Pfizer.

Disclosures. K. M. Shea, Pfizer, Inc: Consultant and Grant Investigator Consulting fee and Grant recipient; S. I. Pelton, Pfizer: Board Member and Gran Investigator, Consulting fee, Research grant and Speaker honorarium; Merck vaccines: Board Member, Consulting fee and Speaker honorarium; GSK: Board Member Consulting fee and Speaker honorarium; Sequiris: Board Member, Consulting fee and Speaker honorarium

1494. Antibiotic Prescription Rates in Children < 24 Months Old Following PCV7/ PCV13 Sequential Implementation

Ron Dagan, $\mathrm{MD}^{1}$; Dana Danino, $\mathrm{MD}^{1,2}$; Shalom Ben-Shimol, $\mathrm{MD}^{1,2}$; David Greenberg $\mathrm{MD}^{1,2}$; Amir Sharf, MAEcon ${ }^{3}$ and Noga Givon-Lavi, $\mathrm{PhD}^{1,2}$; ${ }^{1}$ Faculty of Health Sciences, Ben-Gurion University of the Negev, Beer-Sheva, Israel, ${ }^{2}$ Pediatric Infectious Disease Unit, Soroka University Medical Center, Beer-Sheva, Israel, ${ }^{3}$ Department of Economics and Data Analysis, Clalit (HMO) Southern District, Beer-Sheva, Israel

Session: 165. Pneumococcal Immunization and Epidemiology-North America Friday, October 6, 2017: 12:30 PM

Background. PCV7/PCV13 (PCV) implementation markedly impacted on acute respiratory infection rates in young children, and is thus expected to reduce antibiotic use. We conducted a community-wide study to determine the extent of antibiotic prescription rates (APRs) following PCV implementation.

Methods. The study was conducted from July 2005 through June 2016 among all Jewish children $<24 \mathrm{~m}$, insured by the Clalit Health Maintenance Organization (HMO) in southern Israel ( $74 \%$ of all the region's Jewish children; $n=8,483,2005 ; n=13,604$ 2016). All dispensed prescriptions for oral antibiotics at the HMO were recorded and yearly APRs were calculated by antibiotic category. PCV7 and PCV13 were implemented in July 2009 and November 2010 respectively and rapidly reached $90 \%$ coverage for 3 doses. Epidemiological years were from July through June.

Results. Overall, high APRs were seen throughout the study. A total of 226,035 antibiotic prescriptions were dispensed. Overall annual APR means (per 1,000 \pm SD) were $2068.9 \pm 15.2$ and $1841.1 \pm 39.1$ in 2005-2009 and 2013-2016, respectively (11\% reduction; $95 \%$ CI 10-12\%) (Figure 1). Amoxicillin, the most commonly prescribed antibiotic drug ( $60.8 \%$ of all prescriptions) was reduced by $14 \%$ (95\% CI $13-15 \%)$ (Figure 2). Similar reductions were seen for oral cephalosporins and amoxicillin/ clavulanate. However azithromycin increased continuously throughout the study Calculation of linear trends before and after PCV implementation demonstrated a significant change in trends for amoxicillin, oral cephalosporin and total APRs, strongly suggesting a causative role of PCVs. PCV implementation resulted in an overall reduction of 45,320 prescriptions for a cohort of 100,000 children during their first 2 years of life (95\% CI 41,512 to 49,007$)$

Conclusion. A clear and significant change in all APR trends associated with PCV implementation was observed in children $<24$ months old with a baseline high APR This resulted in a marked decline in antibiotic use. Continuous surveillance is needed to determine further trends, including those for specific antibiotic categories. 